\title{
On Some Higher Order Counting Functions for PSL(2,R)
}

\author{
DŽENAN GUŠIĆ \\ University of Sarajevo \\ Faculty of Sciences and Mathematics \\ Department of Mathematics \\ Zmaja od Bosne 33-35, 71000 Sarajevo \\ BOSNIA AND HERZEGOVINA \\ dzenang@pmf.unsa.ba
}

\begin{abstract}
This paper is devoted to some counting functions of level one and level three in the case of quotient space generated by some strictly hyperbolic Fuchsian group and the upper half-plane. Each of the functions is represented as a sum of some explicit part plus the error term. The explicit part is indexed over singularities of the corresponding Selberg zeta function. In particular, the obtained error term is not larger than $O\left(x^{\frac{3}{4}}\right)$. The method applied in this paper follows traditional approach for achieving the error terms in the case of locally symmetric spaces of real rank one. In order to establish an analogy with the classical case, we consider the counting functions dividedby $x$ and $x$,respectively.
\end{abstract}

Key-Words: Counting functions, upper half-plane, Fuchsian groups, error terms

Received: October 3, 2019. Revised: March 5, 2020. Accepted: March 20, 2020. Published: March 31, 2020.

\section{Introduction}

Our notation will be based od [9] and [15].

Let $F$ be a compact Riemann surface of genus $g$ $\geq 2$. We can therefore represent $F$ as a quotient space $\Gamma \backslash H$, where $\Gamma$ is a strictly hyperbolic Fuchsian group and $H$ is the upper half-plane.

Thus, $\Gamma \subseteq P S L(2, \mathbb{R})$.

If $\mathcal{I}$ is the fundamental polygon of $F$, then $\partial \mathcal{I}=$ $\alpha_{1}^{+} \beta_{1}^{+} \alpha_{1}^{-} \beta_{1}^{-} \ldots \alpha_{g}^{+} \beta_{g}^{+} \alpha_{g}^{-} \beta_{g}^{-}$, where the sides $\alpha_{k}^{-}, \alpha_{k}^{+}$ and $\beta_{k}^{-}, \beta_{k}^{+}$are identified in pairs.

We assume that the sides of $\mathcal{I}$ are piecewise smooth.

As it is known, the upper half-plane $H$ comes with the Poincare metric $d s=\underset{y}{|d z|}$, whose corresponding area element is $d \mu(z)=y^{-2} d x d y$.

Note that the Poincare metric has Gaussian curvature $K=-1$ (see, e.g., [18]).

Suppose that $\pi: H \rightarrow F$ is the universal covering map.

By projecting the Poincare metric onto $F$ via $\pi$, $F$ becomes a compact Rirmannian manifold.

Furthermore, by the Gauss-Bonnet theorem (see, e.g., [17])

$$
A=\operatorname{Area}(F)=\mu(\mathcal{I})=4 \pi(g-1) .
$$

Note that the automorphic group $\Gamma$ is determined up to a conjugation in $P S L(2, \mathbb{R})$. However, the
Poincare metric on $F$ is not affected by such conjugations because of the invariance properties of the Poincare metric on $H$ (we assume through the rest of the paper that $F$ carries the Poincare metric). Furthermore, the Gaussian curvature is still $K=-1$.

In [10], the author concluded that the asymptotic distribution of the closed geodesics is highly influenced by the eigenvalues of the Laplace operator $\Delta$ on $F$, i.e., by the eigenvalues for the problem $\Delta f+$ $\lambda f=0$ on $F$.

The Selberg zeta function $\mathrm{Sel}(s)$ for the group $\Gamma$ is an entire function of order 2 , having a sequence of zeros at $0,-1,-2, \ldots$, with the zero at $s=1$ simple, and having additional zeros in the critical strip $0<$ $\operatorname{Re}(s)<1$. The zeros in the critical strip are located at points which are solutions of the equations $s(s-1)$ $=\lambda_{n}$, where $\lambda_{n}$ ranges through the sequence of eigenvalues, omitting $\lambda_{0}=0$, for the problem $\Delta f+\lambda f=$ 0 on $F$.

The multiplicity of such a zero is the same as the multiplicity of the corresponding eigenvalue (see, e.g., [13], [16]).

Note that the detailed description of the locations and the orders of the zeros of $\mathrm{Sel}(s)$ will be given in the sequel.

In [15, p. 245, Th. 2] (see also, [11]), the author derived the following length spectrum.

If $\operatorname{Sel}(s)$ has zeros $\alpha_{1}, \alpha_{2}, \ldots, \alpha_{n}$ in $\left(\begin{array}{l}3 \\ 4\end{array}, 1\right)$, then, there exist constants $c_{1}, c_{2}, \ldots, c_{n}$, such that 


$$
\varphi_{0}(x)=x+c_{1} x^{\alpha_{1}}+\ldots+c_{n} x^{\alpha_{n}}+O\left(x^{\frac{3}{4}}\right)
$$

as $x \rightarrow+\infty$ (the functions $\varphi_{n}(x), n \in \mathbb{N} \cup\{0\}$ are introduced below).

If, however, $\operatorname{Sel}(s)$ has no zeros in $\left(\frac{3}{4}, 1\right)$, then

$$
\varphi_{0}(x)=x+O\left(x^{\frac{3}{4}}\right)
$$

as $x \rightarrow+\infty$.

The main goal of this research is to derive an analogous result for the function $\frac{\varphi_{1}(x)}{x}$, i.e., a weighted form of the corresponding length spectrum.

In our earlier research [1, p. 466, Th. 1], we derived one such result applying quite complex mathematical apparatus. The obtained remainder $O\left(x^{\frac{1}{2}} \log x\right)$, however, was much better than the classical one $O\left(x^{\frac{3}{4}}\right)$.

Motivated by this fact, that a $\varphi_{1}$ analogue of the classical length spectrum yields a better result, we give yet another proof of Theorem 1 [1] based on application of much simpler mathematical techniques.

\section{Preliminaries}

We adopt the functions $\varphi_{0}(x)$ and $\varphi_{n}(x)$ from $[15$, p. 245].

The first one is given as a sum indexed over $\Gamma_{\text {hyp }}$ with $\operatorname{Norm}\left(\gamma_{0}\right) \leq x$, where $\Gamma_{\text {hyp }}$ denotes the set of $\Gamma$-conjugacy classes of hyperbolic elements in $\Gamma, \operatorname{Norm}\left(\gamma_{0}\right)=\exp \left(\operatorname{len}\left(G_{e o_{\gamma_{0}}}\right)\right)$, len $\left(G_{e o} \gamma_{0}\right)$ is the length of the prime geodesic $\mathrm{Geo}_{\gamma_{0}}$ associated to the conjugacy class $\gamma_{0}$ (it is known fact that prime geodesics over $F$ correspond to the conjugacy classes of primitive hyperbolic elements in $\Gamma$ ). More precisely, if $\gamma \in \Gamma_{h y p}$, then $\gamma$ is an exponent of some primitive $\gamma_{0}$, with the degree $j(\gamma) \in \mathbb{N}$.

For such $\gamma, \Phi(\gamma)$ is defined by $\Phi(\gamma)=$ len $\left(\mathrm{Geo}_{\gamma_{0}}\right)$.

The functions $\varphi_{n}(x), n \in \mathbb{N}$ are defined inductively.

Through the rest of the paper, we shall assume that $l$ is a number, $l \in \mathbb{N}$, and that $d$ and $T$ are constants which will be fixed later.

For a function $f(x)$, we define $\mathcal{D}_{l-1,+}$ by

$$
\begin{aligned}
& \mathcal{D}_{l-1,+} f(x) \\
= & f(x+(l-1) d)-(l-1) f(x+(l-2) d) \\
& +\frac{(l-1)(l-2)}{2} f(x+(l-3) d)-\ldots \\
& +(-1)^{l-1} f(x) .
\end{aligned}
$$

By $[3$, p. $315,(22)], \mathcal{D}_{l-1,+} f(x)$ can be represented as an iterated integral (if $f$ is a differentiable function of appropriate order).

Thus, for some $x \leq \tilde{x} \leq x+(l-1) d$, an analogue of the estimate (23) in [3] holds also true.

The number of zeros of $\mathrm{Sel}(s)$ on the critical line will be denoted by $\mathfrak{n}(t)$. as $\frac{A}{4 \pi} t^{2}$

We shall apply the fact that $\mathfrak{n}(t)$ can be estimated

\section{Main result}

We shall prove the following theorem.

Theorem 1. Let $F$ be a compact Riemann surface of genus $g \geq 2$. Then,

$$
\begin{aligned}
& \frac{\varphi_{1}(x)}{x} \\
= & \frac{1}{2} x+\sum_{\substack{s_{0} \in S_{0, \mathbb{R}} \\
\frac{1}{2}<s_{0}<1}} \text { ord }\left(s_{0}, 0\right) s_{0}^{-1}\left(s_{0}+1\right)^{-1} x^{s_{0}} \\
& +O\left(x^{\frac{1}{2}} \log x\right)
\end{aligned}
$$

as $x \rightarrow+\infty$, where $S_{0, \mathbb{R}}$ is the set of zeros of $\operatorname{Sel}(s)$ such that $s_{0} \in(0,1)$, and ord $\left(s_{0}, 0\right)$ is the multiplicity of $s_{0}$.

Proof. By $\left[15\right.$, p. 245, Th. 1.'], $\varphi_{l}(x)$ can be represented as the sum $\sum_{p=0}^{1}(-1)^{p} W_{p}$, where $W_{p}$ is the sum $\sum_{z \in A_{p, l}} a_{z, p, l}$

Here, $A_{p, l}$ denotes the set of poles of the corresponding function, and $a_{z, p, l}$ 's are the attached residues.

In general, we may point out that the Selberg zeta function $\operatorname{Sel}(s+p)$ has a zero at $1-p$ of order 1 , a zero at $0-p$ of order $2 g-1$, zeros at $-k-p, k \in$ $\{1,2, \ldots\}$, whose orders are $(2 g-2)(2 k+1)$. 
The non-trivial zeros of $\operatorname{Sel}(s+p)$ are all contained in the union of the interval $(0-p, 1-p)$ with the vertical line $\frac{1}{2}-p+\mathrm{i} \mathbb{R}$, i.e., in $(-p, 1-p) \cup$ $\left(\frac{1}{2}-p+\mathrm{i} \mathbb{R}\right)$.

As we already noted, the values $0,-1, \ldots,-l$ are zeros of $\operatorname{Sel}(s+p)$. Hence, these values are poles of order two of the corresponding function.

The values $-(l+1),-(l+2), \ldots$ are then the simple poles of the same function.

Finally, the value 1 is a simple pole if $p=0$.

The set of zeros $s_{p}$ of $\operatorname{Sel}(s+p)$ such that $s_{p} \notin$ $\mathbb{Z}$ will be denoted by $S_{p}$.

In other words, $S_{p} \subseteq(-p, 1-p) \cup$ $\left(\frac{1}{2}-p+\mathrm{i} \mathbb{R}\right)$.

Note that the values $s_{p}$, where $s_{p} \in S_{p}$ are also simple poles of the function in the case at hand. $A_{p, l}$.

Now, we determine the residues $a_{z, p, l}$ 's for $z \in$

Let $z$ be a zero of $\operatorname{Sel}(s+p)$ of multiplicity ord $(z, p)$.

Furthermore, let $q_{i}(z, p)$ 's be the corresponding coefficients in the expansion of the logarithmic derivative of $\operatorname{Sel}(s+p)$ near $z$.

If $z \in A_{p, l}$, and $z=s_{p} \in S_{p}$, then $a_{s_{p}, p, l}$ 's are calculated in the same way as in [3, p. 314, (13)].

Suppose that $z \in A_{p, l}$, and $z=-j \in$ $\{0,-1, \ldots,-l\}$.

For the final form of the corresponding residue $a_{-j, p, l}$ in this case, we refer to [3, (14)].

Now, suppose that $z \in A_{p, l}, z=-j \in$ $\{-(l+1),-(l+2), \ldots\}$.

In this case, $a_{-j, p, l}$ is given by ord $(-j, p) \prod_{q=0}^{l}(-j+q)^{-1} x^{-j+l}$. $\frac{x^{1+l}}{(l+1) !}$

Furthermore, if $z \in A_{0, l}, z=1$, then $a_{1,0, l}=$

We shall consider the following subsets of the set $S_{p}: S_{p, \mathbb{R}}=S_{p} \cap \mathbb{R}$, and $S_{p, \frac{1}{2}-p}=S_{p} \backslash S_{p, \mathbb{R}}$ for $p \in$ $\{0,1\}$. $i \mathbb{R}$.

Thus, $S_{p, \mathbb{R}} \subseteq(-p, 1-p)$ and $S_{p, \frac{1}{2}-p} \subseteq \frac{1}{2}-p+$

Assume that $z \in A_{p, l}$, and $z \in S_{p, \frac{1}{2}-p}$.

By the very definition of the operator $\mathcal{D}_{l-1,+}$, it immediately follows that $d^{-(l-1)} \mathcal{D}_{l-1,+} a_{z, p, l}$ can be estimated by $O\left(d^{-(l-1)}|z|^{-l-1} x^{\frac{1}{2}+l}\right)$

On the other side, an application of the mean value theorem, yields that

$|\operatorname{ord}(z, p)||z|^{-1}|z+1|^{-1}\left(x+\sum_{i=2}^{l} q_{i}\right)^{\frac{1}{2}-p+1}$ dominates $\left|d^{-(l-1)} \mathcal{D}_{l-1,+} a_{z, p, l}\right|$ for some $0 \leq q_{i} \leq d, i$ $\in\{2,3, \ldots, l\}$.
Thus, $O\left(|z|^{-2} x^{\frac{3}{2}}\right)$ is the second estimate for $d^{-(l-1)} \mathcal{D}_{l-1,+} a_{z, p, l}$.

Having in mind these two estimates, one easily concludes that the sum $\sum_{z \in S_{p, \frac{1}{2}-p}} d^{-(l-1)} \mathcal{D}_{l-1,+} a_{z, p, l}$, and hence the $\operatorname{sum} \sum_{p=0}^{1}(-1)^{p} \sum_{z \in S_{p, \frac{1}{2}-p}} d^{-(l-1)} \mathcal{D}_{l-1,+} a_{z, p, l}$ are $O\left(x^{\frac{3}{2}} \log T\right)+O\left(d^{-(l-1)} x^{\frac{1}{2}+l} T^{-l+1}\right)$.

Furthermore, $\frac{1}{2} x^{2}+O(d x)+O\left(d^{2}\right)$ dominates $d^{-(l-1)} \mathcal{D}_{l-1,+} a_{1,0, l}$.

Suppose that $d=x^{\alpha}(\log x)^{\beta}$ and $T=$ $x^{\gamma}(\log x)^{\delta}$.

Then,

$$
\begin{aligned}
d^{2}= & x^{2 \alpha}(\log x)^{2 \beta}, \\
& x^{\frac{3}{2}} \log T \\
= & \gamma x^{\frac{3}{2}} \log x+\delta x^{\frac{3}{2}} \log \log x, \\
& d^{-l+1} x^{\frac{1}{2}+l} T^{-l+1} \\
= & x^{(-l+1) \alpha+\frac{1}{2}+l+(-l+1) \gamma}(\log x)^{(-l+1) \beta+(-l+1) \delta} .
\end{aligned}
$$

Since $\log \log x$ is dominated by $\log x$, we want that

$$
\begin{aligned}
& 2 \alpha=\frac{3}{2}=(-l+1) \alpha+\frac{1}{2}+l+(-l+1) \gamma, \\
& 2 \beta=1=(-l+1) \beta+(-l+1) \delta .
\end{aligned}
$$

It is not so hard to conclude that $\alpha=\frac{3}{4}, \beta=\frac{1}{2}$, $\gamma=\frac{1}{4}$ and $\delta=\frac{1+l}{2(1-l)}$, i.e., $d=x^{\frac{3}{4}}(\log x)^{\frac{1}{2}}$ and $T=$ $x^{\frac{1}{4}}(\log x)^{\frac{1+l}{2(1-l)}}$.

Since, in this case, $O(d x)=O\left(x^{\frac{7}{4}}(\log x)^{\frac{1}{2}}\right)$, it follows that the aforementioned remainders are all $O\left(x^{\frac{7}{4}}(\log x)^{\frac{1}{2}}\right)$.

Next, we consider

$$
\begin{aligned}
d x= & x^{\alpha+1}(\log x)^{\beta}, \\
& x^{\frac{3}{2}} \log T \\
= & \gamma x^{\frac{3}{2}} \log x+\delta x^{\frac{3}{2}} \log \log x, \\
& d^{-l+1} x^{\frac{1}{2}+l} T^{-l+1} \\
= & x^{(-l+1) \alpha+\frac{1}{2}+l+(-l+1) \gamma}(\log x)^{(-l+1) \beta+(-l+1) \delta} .
\end{aligned}
$$


It follows that,

Reasoning in the same way as in the previous case, we are interested in

$$
\begin{aligned}
\alpha+1 & =\frac{3}{2}=(-l+1) \alpha+\frac{1}{2}+l+(-l+1) \gamma, \\
\beta & =1=(-l+1) \beta+(-l+1) \delta .
\end{aligned}
$$

Thus, $\alpha=\frac{1}{2}, \beta=1, \gamma=\frac{1}{2}$ and $\delta=\frac{l}{1-l}$, that is, $d=x^{\frac{1}{2}} \log x$ and $T=x^{\frac{1}{2}}(\log x)^{\frac{l}{(1-l)}}$.

Also, in this case, the error term $O\left(d^{2}\right)$ is $O\left(x(\log x)^{2}\right)$.

Consequently, the error terms given above are all $O\left(x^{\frac{3}{2}} \log x\right)$.

Since the equality $O(d x)=O\left(d^{2}\right)$ yields at least the error term $O\left(x^{2}\right)$, the remaining two cases are not interesting for our research.

The discussion conducted above, yields that $O\left(x^{\frac{3}{2}} \log x\right)$ is the remainder we are looking for, and is established for $d=x^{\frac{1}{2}} \log x$ and $T=$ $x^{\frac{1}{2}}(\log x)^{\frac{l}{(1-l)}}$.

The sum over $s_{p} \in S_{p, \mathbb{R}}, \frac{1}{2}<s_{p}<1, p \in\{0,1\}$, is obviously equal to the sum over $s_{0} \in S_{0, \mathbb{R}}, \frac{1}{2}<s_{0}$ $<1$.

Thus, for $d=x^{\frac{1}{2}} \log x$, the sum is equal to

$$
\begin{aligned}
& \sum_{\substack{s_{0} \in S_{0, \mathbb{R}} \\
\frac{1}{2}<s_{0}<1}} \operatorname{ord}\left(s_{0}, 0\right)\left(s_{0}\right)^{-1}\left(s_{0}+1\right)^{-1} x^{s_{0}+1} \\
& +O\left(x^{\frac{3}{2}} \log x\right) .
\end{aligned}
$$

A we noted earlier, $\operatorname{Sel}(s+p)$ has zeros at $-k-$ $p, k \in\{1,2, \ldots\}$ of orders $(2 g-2)(2 k+1)$.

In particular, $\mathrm{Sel}(s)$ has zeros at $-k$, $k \in\{1,2, \ldots\}$ of orders $(2 g-2)(2 k+1)$, while $\operatorname{Sel}(s+1)$ has zeros at $-k-1, k \in\{1,2, \ldots\}$ of orders $(2 g-2)(2 k+1)$.

Moreover, we denoted by $\operatorname{ord}(z, 0)$ the multiplicity of the zero $z$ of $\operatorname{Sel}(s)$.

In particular, ord $(z, 1)$ is the multiplicity of the zero $z$ of $\operatorname{Sel}(s+1)$.

Thus, ord $(-k, 0)=(2 g-2)(2 k+1), k \in$ $\{1,2, \ldots\}$, and $\operatorname{ord}(-k-1,1)=\operatorname{ord}(-(k+1), 1)$ $=(2 g-2)(2 k+1), k \in\{1,2, \ldots\}$.

Hence, ord $(-j, 0)=(2 g-2)(2 j+1)$, ord $(-j, 1)=\operatorname{ord}(-(j-1)-1,1)=$ $(2 g-2)(2(j-1)+1)=(2 g-2)(2 j-1)$.

$$
\begin{aligned}
& \sum_{p=0}^{1}(-1)^{p} \sum_{j=l+1}^{+\infty} a_{-j, p, l} \\
= & (2 g-2) \sum_{j=l+1}^{+\infty} \frac{2 j+1}{(-j)(-j+1) \ldots(-j+l)} x^{-j+l}- \\
& (2 g-2) \sum_{j=l+1}^{+\infty} \frac{2 j-1}{(-j)(-j+1) \ldots(-j+l)} x^{-j+l} \\
= & (-1)^{l+1}(4 g-4) \sum_{j=l+1}^{+\infty} \frac{1}{j(j-1) \ldots(j-l)} x^{-j+l} .
\end{aligned}
$$

Thus,

$$
\begin{aligned}
& \left|\sum_{p=0}^{1}(-1)^{p} \sum_{j=l+1}^{+\infty} a_{-j, p, l}\right| \\
= & (4 g-4) \sum_{j=l+1}^{+\infty} \frac{1}{j(j-1) \ldots(j-l)} x^{-j+l} \\
\leq & (4 g-4) x^{-1} \sum_{j=l+1}^{+\infty} \frac{1}{j(j-1) \ldots(j-l)} .
\end{aligned}
$$

Since the last series converges, we conclude that the sum of $a_{-j, p, l}$ 's over $j \in\{l+1, l+2, \ldots\}, p \in$ $\{0,1\}$ is $O\left(x^{-1}\right)$.

If we apply $\mathcal{D}_{l-1,+}$ to this sum, the remainder $O\left(x^{-1}\right)$ will not be changed.

Consequently, for $d=x^{\frac{1}{2}} \log x$, the error term $O\left(x^{-1}\right)$ multiplied by $d^{-(l-1)}$ gives us

$$
\begin{aligned}
& O\left(\left(x^{\frac{1}{2}} \log x\right)^{-l+1} x^{-1}\right) \\
= & O\left(x^{-\frac{1}{2}-\frac{l}{2}}(\log x)^{-l+1}\right) \\
= & O\left(x^{-\frac{3}{2}}(\log x)^{-1}\right) .
\end{aligned}
$$

Since

$$
\begin{aligned}
& \left|\sum_{p=0}^{1}(-1)^{p} \sum_{j=2}^{l} d^{-(l-1)} \mathcal{D}_{l-1,+} a_{-j, p, l}\right| \\
\leq & x^{-1} \sum_{p=0}^{1} \sum_{j=2}^{l}|o(-j, p, l)|,
\end{aligned}
$$

where the constant $o(-j, p, l)$ is given by 


$$
\begin{aligned}
& o(-j, p, l) \\
= & (-1)^{j-2} \text { ord }(-j, p)(l-j) !(j-2) ! \times \\
& \times \prod_{\substack{q=0 \\
q \neq j}}^{l}(-j+q)^{-1},
\end{aligned}
$$

it follows that the corresponding sum is estimated by $O\left(x^{-1}\right)$.

Reasoning in the same way as above (applying the main properties of $\mathcal{D}_{l-1,+}$ ), we obtain that the sum corresponding to $a_{-1, p, l}, p \in\{0,1\}$ resp. the sum corresponding to $a_{0, p, l}, p \in\{0,1\}$, is $O(\log x)$ resp. $O(x \log x)$.

Note that the sum over $s_{p} \in S_{p, \mathbb{R}},-1<s_{p}<0, p$ $\in\{0,1\}$ resp. the sum over $s_{p} \in S_{p, \mathbb{R}}, 0<s_{p} \leq \frac{1}{2}, p$ $\in\{0,1\}$, is actually the sum over $s_{1} \in S_{1, \mathbb{R}},-1<s_{1}$ $<0$ resp. the sum over $s_{0} \in S_{0, \mathbb{R}}, 0<s_{0} \leq \frac{1}{2}$.

These two sums, however, are $O(x)$ and $O\left(x^{\frac{3}{2}}\right)$, respectively.

Having in mind the subsets of $A_{p, l}$ we have considered above, we may write

$$
\begin{aligned}
& d^{-(l-1)} \mathcal{D}_{l-1,+} \varphi_{l}(x) \\
& =d^{-(l-1)} \mathcal{D}_{l-1,+} a_{1,0, l}+ \\
& \sum_{p=0}^{1}(-1)^{p} \sum_{z \in S_{p, \frac{1}{2}-p}} d^{-(l-1)} \mathcal{D}_{l-1,+} a_{z, p, l}+ \\
& \sum_{p=0}^{1}(-1)^{p} \sum_{\substack{s_{p} \in S_{p, \mathbb{R}} \\
\frac{1}{2}<s_{p}<1}} d^{-(l-1)} \mathcal{D}_{l-1,+} a_{s_{p}, p, l}+ \\
& d^{-(l-1)} \mathcal{D}_{l-1,+} \sum_{p=0}^{1}(-1)^{p} \sum_{j=l+1}^{+\infty} a_{-j, p, l}+ \\
& \sum_{p=0}^{1}(-1)^{p} \sum_{j=2}^{l} d^{-(l-1)} \mathcal{D}_{l-1,+} a_{-j, p, l}+ \\
& \sum_{p=0}^{1}(-1)^{p} d^{-(l-1)} \mathcal{D}_{l-1,+} a_{-1, p, l}+ \\
& \sum_{p=0}^{1}(-1)^{p} d^{-(l-1)} \mathcal{D}_{l-1,+} a_{0, p, l}+ \\
& \sum_{p=0}^{1}(-1)^{p} \sum_{\substack{s_{p} \in S_{p, \mathbb{R}} \\
-1<s_{p}<0}} d^{-(l-1)} \mathcal{D}_{l-1,+} a_{s_{p}, p, l}+
\end{aligned}
$$

$$
\sum_{p=0}^{1}(-1)^{p} \sum_{\substack{s_{p} \in S_{p, \mathbb{R}} \\ 0<s_{p} \leq \frac{1}{2}}} d^{-(l-1)} \mathcal{D}_{l-1,+} a_{s_{p}, p, l}
$$

Taking into account that $d^{-(l-1)} \mathcal{D}_{l-1,-} \varphi_{l}(x)$ is not larger than $\varphi_{1}(x)$, and the fact that $\varphi_{1}(x)$ is not larger than $d^{-(l-1)} \mathcal{D}_{l-1,+} \varphi_{l}(x)\left(\mathcal{D}_{l-1,-}\right.$ is defined in [14] in a similar way), it follows that for $d=x^{\frac{1}{2}} \log x$ and $T=x^{\frac{1}{2}}(\log x)^{\frac{l}{1-l}}$

$$
\begin{aligned}
& \varphi_{1}(x) \\
& =\frac{1}{2} x^{2}+\sum_{\substack{s_{0} \in S_{0, \mathbb{R}} \\
\frac{1}{2}<s_{0}<1}} \operatorname{ord}\left(s_{0}, 0\right) s_{0}^{-1}\left(s_{0}+1\right)^{-1} x^{s_{0}+1} \\
& \\
& \quad+O\left(x^{\left.\frac{3}{2} \log x\right) .}\right.
\end{aligned}
$$

Thus,

$$
\begin{aligned}
& \frac{\varphi_{1}(x)}{x} \\
= & \frac{1}{2} x+\sum_{\substack{s_{0} \in S_{0, \mathbb{R}} \\
\frac{1}{2}<s_{0}<1}} \operatorname{ord}\left(s_{0}, 0\right) s_{0}^{-1}\left(s_{0}+1\right)^{-1} x^{s_{0}} \\
& +O\left(x^{\frac{1}{2}} \log x\right) .
\end{aligned}
$$

This completes the proof.

\section{Case $\varphi_{3}(x)$}

In this section we shall consider the function $\varphi_{3}(x)$. We shall apply the main properties of the operator $\mathcal{D}_{l-3,+} f(x)$, where $f(x)$ is some function.

In particular, if $z \in A_{p, l}$, and $z \in S_{p, \frac{1}{2}-p}$, then, by the definition of $\mathcal{D}_{l-3,+}$, it follows that $d^{-l+3} \mathcal{D}_{l-3,+} a_{z, p, l}$ is $O\left(d^{-l+3}|z|^{-l-1} x^{\frac{1}{2}+l}\right)$.

Furthermore, if we apply the fact that $d^{-l+3} \mathcal{D}_{l-3,+} a_{z, p, l}$ can be represented as an iterated integral of some differentiable function of appropriate order, then, we obtain that $d^{-l+3} \mathcal{D}_{l-3,+} a_{z, p, l}$ is bounded by $O\left(|z|^{-4} x^{\frac{7}{2}}\right)$.

Thus, combining these two estimates, we conclude that the sum of the elements $d^{-l+3} \mathcal{D}_{l-3,+} a_{z, p, l}$ (over $p \in\{0,1\}$ and $z \in S_{p, \frac{1}{2}-p}$ ) is determined by the sum $O\left(x^{\frac{7}{2}} T^{-2}\right)+O\left(d^{-l+3}|z|^{-l-1} T^{-l+1}\right)$, where 
$T$ is a constant that will be fixed later (the constant $d$ will also be fixed later, but, for now, it is assumed that $d$ is dominated by $O(x)$ ).

Now we apply the operator $d^{-l+3} \mathcal{D}_{l-3,+}$ to the residue $a_{1,0, l}$ whose position is on the right.

If we use the fact that $d^{-l+3} \mathcal{D}_{l-3,+}$ applied to some differentiable function $f(x)$ (of appropriate order) is $f^{(l-3)}(\tilde{x})$ for some $x \leq \tilde{x} \leq x+(l-3) d$, then, we obtain that $d^{-l+3} \mathcal{D}_{l-3,+} a_{1,0, l}$ is given by $\frac{1}{4 !} x^{4}+O\left(x^{3} d\right)+O\left(x^{2} d^{2}\right)+O\left(x d^{3}\right)+O\left(d^{4}\right)$.

One should be interested to determine $d$ and $T$ such that the error term $O\left(x^{3} d\right)$, and the error terms $O\left(x^{\frac{7}{2}} T^{-2}\right), O\left(d^{-l+3}|z|^{-l-1} T^{-l+1}\right)$ be the same.

As it is usual, denoting $d$ and $T$ by some $x^{\alpha}$ and $x^{\beta}$, respectively, we easily conclude that $3+\alpha, \frac{7}{2}$ $-2 \beta$ and $-\alpha(l-3)+\frac{1}{2}+l+\left(\frac{1}{4}-\frac{1}{2} \alpha\right)(-l+1)$ must be the same.

Solving this system, we obtain that $\alpha=\frac{3}{2}$ and $\beta=$ $-\frac{1}{2}$. In other words $d$ is not bounded by $O(x)$, which means that the calculations obtained previously could not be valid in this case. Hence, we don't consider this case anymore.

For our needs, it will be sufficient to determine $d$ and $T$ such that $O\left(d^{4}\right)$, and the error terms $O\left(x^{\frac{7}{2}} T^{-2}\right), O\left(d^{-l+3}|z|^{-l-1} T^{-l+1}\right)$ be the same.

Proceeding in the same way as in the previous case, we obtain that $4 \alpha$, and $\frac{7}{2}-2 \beta,-\alpha(l-3)+$ $\frac{1}{2}+l+\left(\frac{7}{4}-2 \alpha\right)(-l+1)$ must be the same. It follow that $\alpha=\frac{3}{4}$ and $\beta=\frac{1}{4}$. Hence, the condition that $d$ is dominated by $O(x)$ is provided. Thus, the $O-$ terms: $O\left(x^{3} d\right), O\left(x^{2} d^{2}\right), O\left(x d^{3}\right), O\left(d^{4}\right)$ are all dominated by $O\left(x^{3} d\right)$. This error term, however, is given now by $O\left(x^{\frac{15}{4}}\right)$.

We may say now that we are interested in achieving the error term $O\left(x^{\frac{15}{4}}\right)$ in the final form of the corresponding weighted prime geodesic theorem.

Now, we consider the sum of the elements $d^{-l+3} \mathcal{D}_{l-3,+} a_{s_{p}, p, l}$ along $p \in\{0,1\}$ and $s_{p} \in S_{p, \mathbb{R}}, 0$ $<s_{p}<1$. It is not so hard to conclude that this sum is given by

$$
\begin{aligned}
& \sum_{\substack{s_{0} \in S_{0, \mathbb{R}} \\
0<s_{0}<1}} \operatorname{ord}\left(s_{0}, 0\right) s_{0}^{-1}\left(s_{0}+1\right)^{-1} \times \\
& \times\left(s_{0}+2\right)^{-2}\left(s_{0}+3\right)^{-1} x^{s_{0}+3}+O\left(x^{3} d\right) .
\end{aligned}
$$

Next, we consider the corresponding sum over $p$ $\in\{0,1\}$ and $j \in\{l+1, l+2, \ldots\}$.

We shall apply a modified method of the method given in the previous sections. Thus, we obtain that the sum in the case at hand is actually

$$
\begin{aligned}
& (2 g-2) \sum_{j=l+1}^{+\infty} \frac{2 j+1}{j(j-1) \ldots(j-3) \tilde{x}_{-j, 0, l}^{j-3}}- \\
& (2 g-2) \sum_{j=l+1}^{+\infty} \frac{2 j-1}{j(j-1) \ldots(j-3) \tilde{x}_{-j, 1, l}^{j-3}}
\end{aligned}
$$

for some $x \leq \tilde{x}_{-j, p, l} \leq x+(l-3) d$.

Now, it follows that

$\left|\sum_{p=0}^{1}(-1)^{p} \sum_{j=l+1}^{+\infty} d^{-l+3} \mathcal{D}_{l-3,+} a_{-j, p, l}\right|$ is bounded

$$
\frac{4(g-1)}{(l-1)(l-2) x^{l-2}} \text {. }
$$

Thus, the corresponding sum is $O\left(\frac{1}{x^{l-2}}\right)$.

The next sum is the sum over $p \in\{0,1\}$ and $j \in$ $\{4,5, \ldots, l\}$.

The constant $o(-j, p, l)$ from above is modified accordingly, and is

$$
\begin{aligned}
o(-j, p, l) \\
=(-1)^{j-4} \operatorname{ord}(-j, p)(l-j) !(j-4) ! \times \\
\times \prod_{\substack{q=0 \\
q \neq j}}^{l}(-j+q)^{-1} .
\end{aligned}
$$

Thus, the sum

$$
\sum_{p=0}^{1} \sum_{j=4}^{l}|o(-j, p, l)| \frac{1}{x^{j-3}}
$$

is $O\left(x^{-1}\right)$.

Furthermore, the sum over $p \in\{0,1\}$ of $d^{-l+3} \mathcal{D}_{l-3,+} a_{-3, p, l}$ is $O(\log x)$.

The sum of the elements $d^{-l+3} \mathcal{D}_{l-3,+} a_{-2, p, l}$ along $p \in\{0,1\}$ is $O(x \log x)$.

Finally, the corresponding sum of $d^{-l+3} \mathcal{D}_{l-3,+} a_{-1, p, l}$ is $O\left(x^{2} \log x\right)$.

Reasoning in the same way as above, we conclude that

$$
\sum_{p=0}^{1}(-1)^{p} d^{-l+3} \mathcal{D}_{l-3,+} a_{0, p, l}=O\left(x^{3} \log x\right) .
$$

The last sum we have to consider is the sum of the elements $d^{-l+3} \mathcal{D}_{l-3,+} a_{s_{p}, p, l}$ along $p \in\{0,1\}$ and 
$s_{p} \in S_{p, \mathbb{R}},-1<s_{p}<0$. Obviously, this sum is the minus sum of the elements $d^{-l+3} \mathcal{D}_{l-3,+} a_{s_{1}, 1, l}$ over $s_{1} \in S_{1, \mathbb{R}},-1<s_{1}<0$. The last sum, however, is $O\left(x^{3}\right)$.

Combining the estimates derived above, we conclude that $\varphi_{3}(x)$ is not larger than

$$
\begin{aligned}
& \frac{1}{4 !} x^{4}+\sum_{\substack{s_{0} \in S_{0, \mathbb{R}} \\
0<s_{0}<1}} \operatorname{ord}\left(s_{0}, 0\right) s_{0}^{-1}\left(s_{0}+1\right)^{-1} \times \\
& \times\left(s_{0}+2\right)^{-2}\left(s_{0}+3\right)^{-1} x^{s_{0}+3}+O\left(x^{3} d\right)+ \\
& O\left(x^{\frac{7}{2}} T^{-2}\right)+O\left(d^{-l+3}|z|^{-l-1} T^{-l+1}\right)+ \\
& O\left(x^{3} \log x\right) .
\end{aligned}
$$

Hence, putting $d$ and $T$ to be as we derived above $\left(x^{\frac{3}{4}}\right.$ and $x^{\frac{1}{4}}$ ), it follows that $\varphi_{3}(x)$ is not larger than

$$
\begin{aligned}
& \frac{1}{4 !} x^{4}+\sum_{\substack{s_{0} \in S_{0, \mathbb{R}} \\
0<s_{0}<1}} \operatorname{ord}\left(s_{0}, 0\right) s_{0}^{-1}\left(s_{0}+1\right)^{-1} \times \\
& \times\left(s_{0}+2\right)^{-2}\left(s_{0}+3\right)^{-1} x^{s_{0}+3}+O\left(x^{\frac{15}{4}}\right) .
\end{aligned}
$$

Similarly, one concludes that the last sum in not larger than $\varphi_{3}(x)$.

Thus, we have proved the following theorem.

Theorem 2. Let $F$ be a compact Riemann surface of genus $g \geq 2$. Then, $\frac{1}{x^{3}} \varphi_{3}(x)$ is

$$
\begin{aligned}
& \frac{1}{4 !} x+\sum_{\substack{s_{0} \in S_{0, \mathbb{R}} \\
\frac{3}{4}<s_{0}<1}} \operatorname{ord}\left(s_{0}, 0\right) s_{0}^{-1}\left(s_{0}+1\right)^{-1} \times \\
& \times\left(s_{0}+2\right)^{-2}\left(s_{0}+3\right)^{-1} x^{s_{0}}+O\left(x^{\frac{3}{4}}\right)
\end{aligned}
$$

as $x \rightarrow+\infty$.

\section{Remarks}

In order to derive (1), we first proved that the sum of $a_{-j, p, l}$ 's over $j \in\{l+1, l+2, \ldots\}, p \in\{0,1\}$, is $O\left(x^{-1}\right)$.

Note that one could also use the fact that $d^{-(l-1)} \mathcal{D}_{l-1,+} a_{-j, p, l}$ is

ord $(-j, p)(-j)^{-1}(-j+1)^{-1} \tilde{x}_{-j, p, l}^{-j+1}$ for some $x \leq$ $\tilde{x}_{-j, p, l} \leq x+(l-1) d$, to directly estimate the sum

$$
\sum_{p=0}^{1}(-1)^{p} \sum_{j=l+1}^{+\infty} d^{-(l-1)} \mathcal{D}_{l-1,+} a_{-j, p, l}
$$

In [1], the authors also derived a weighted length spectrum for real hyperbolic manifolds with cusps. Such research represents a generalization of the researches conducted in [14], [2].

A weighted form of the result obtained in [3] (see also, [6]) is derived in [7].

For yet another proof of the main result in [15], we refer to [8].

Note that the author in [19] also considered a weighted length spectrum. His object of research were the automorphic $L$-functions.

A lot of useful ideas and calculations the author applied in this research are adopted from [4], [5] and [12].

\section{References:}

[1] M. Avdispahić and Dž. Gušić, A Weighted Prime Geodesic Theorem, Mathematica Balkanica 25, 2011, pp. 463-474.

[2] M. Avdispahić and Dž. Gušić, On the error term in the prime geodesic theorem, Bull. Korean Math. Soc. 49, 2012, pp. 367-372.

[3] M. Avdispahić and Dž. Gušić, On the length spectrum for compact locally symmetric spaces of real rank one, WSEAS Trans. on Math. 16, 2017, pp. 303-321.

[4] R. P. Boas, Entire Functions, Academic Press Inc., Publishers, New York 1954

[5] J. B. Conway, Functions of One Complex Variable, Springer-Verlag, New York-HeidelbergBerlin 1978

[6] Dž. Gušić, Prime geodesic theorem for compact even - dimensional locally symmetric Riemannian manifolds of strictly negative sectional curvature, WSEAS Trans. on Math. 17, 2018, pp. 188-196.

[7] Dž. Gušić, A Weighted Generalized Prime Geodesic Theorem, WSEAS Trans. on Math. 17, 2018, pp. 237-251.

[8] Dž. Gušić, Prime Geodesic Theorem for Compact Riemann Surfaces, Int. J. on Circuits, Systems and Signal Processing 13, 2019, pp. 747753.

[9] D. Hejhal, The Selberg trace formula for PSL $(2, \mathbb{R})$. Vol. I. Lecture Notes in Mathematics 548, Springer-Verlag, Berlin-Heidelberg 1976 
[10] H. Huber, Zur analytischen Theorie hyperbolischer Raumformen und Bewegungsgruppen. II, Math. Annalen 142, 1961, pp. 385-398.

[11] H. Huber, Zur analytischen Theorie hyperbolischer Raumformen und Bewegungsgruppen. II (Nachtrag zu Math. Annalen 142, 385-398, 1961), Math. Annalen 143, 1961, pp. 463-464.

[12] A. E. Ingham, The Distribution of Prime Numbers, Cambridge Mathematical Library, 1990

[13] H. P. McKean, Selberg's trace formula as applied to a compact Riemann surface, Comm. Pure Appl. Math. 25, 1972, pp. 225-246.

[14] J. Park, Ruelle zeta function and prime geodesic theorem for hyperbolic manifolds with cusps, in: G. van Dijk, M. Wakayama (eds.), Casimir force, Casimir operators and Riemann hypothesis, de Gruyter, Berlin 2010, pp. 89-104.
[15] B. Randol, On the asymptotic distributon of closed geodesics on compact Riemann surfaces, Trans. Amer. Math. Soc. 233, 1977, pp. 241-247.

[16] A. Selberg, Harmonic analysis and discontinuous groups in weakly symmetric Riemannian spaces with applications to Diriclet series, J. Indian Math. 20, 1956, pp. 47-87.

[17] C. L. Siegel, Topics in Complex Function Theory, Wiley-Interscience, 1971

[18] J. J. Stocker, Differential Geometry, Wiley-Interscience, 1969

[19] H. Tang, The Generalized Prime Number Theorem for Automorphic L-Functions, Chin. Ann. Math. 3, 2009, pp. 251-260. 